\title{
The use of YouTube for the self-management of arthritis and joint pain: a patient and public involvement study
}

Osman Ahmed, Sophie L Peters, Carol S Bond, Hopin Lee, Mark Merolli, Jem Lawson, Jo Adams

Background: A growing number of individuals are obtaining health information online. This method of information acquisition supports individuals with chronic health conditions (such as arthritis) in self-managing their symptoms. One of these online sources is YouTube, the second most visited website in the world. This video sharing platform hosts a variety of health information that is accessed by the public. Given its popularity and user-created content, YouTube has potential to be a powerful health information dissemination tool. However, the most frequently accessed arthritis-related videos on YouTube are not created by registered healthcare professionals, and YouTube has been reported to provide insufficient information into the management of hip arthritis. To date, there has been no exploration of the perceptions of people living with arthritis and joint pain on what they view as the most meaningful and timely research agenda for exploring arthritis self-management information on YouTube. The current project's objective was to engage with patient and public involvement (PPI) partners living with joint pain, in order to identify their priorities for research into the use of YouTube for the self-management of joint pain.

Methods: A semi-structured focus group with five PPI partners took place in May 2018. Focus group questions were designed by the research team which included a PPI member. The questions explored the PPI partners' experiences of using YouTube in the management of joint pain and their recommendations regarding further research into the area. The focus group was audio recorded, and later transcribed and analysed using thematic analysis. All data were anonymised and no personal details were included in the results.

Results: PPI partners felt it was important to encourage self-management of joint pain and that YouTube could constitute a useful platform to support this self-management. The partners discussed the reliability of information shared via YouTube, as well as the credibility of the individuals sharing this material. They also identified the limited access to healthcare professionals (GPs and physiotherapists) that persons living with arthritis and joint pain have, and the ways in which this could be addressed using YouTube videos. The outcomes of this study provided a framework for future research priorities. These covered: further exploration into the quality of YouTube information (particularly the quality of content delivered); use of YouTube for self-management of arthritis and joint pain; and its referral to and delivery of YouTube content.

Conclusion: YouTube is frequently accessed for healthcare information, and PPI partners in this study identified the importance of ensuring robust research is conducted. Identified research areas included exploring what information people with arthritis look for on YouTube and what questions GPs are most frequently asked with regards to arthritis, and whether the answers to these questions can be built into content on YouTube. 